\title{
Border states in heterosheets with hexagonal symmetry
}

\author{
Susumu Okada, ${ }^{1}$ Masatsura Igami, ${ }^{1}$ Kyoko Nakada, ${ }^{2}$ and Atsushi Oshiyama ${ }^{3}$ \\ ${ }^{1}$ Institute of Material Science, University of Tsukuba, Tennodai, Tsukuba 305-8573, Japan \\ ${ }^{2}$ College of Science and Engineering, Aoyama Gakuin University, Atsugi 243-0123, Japan \\ ${ }^{3}$ Institute of Physics, University of Tsukuba, Tennodai, Tsukuba 305-8571, Japan
}

(Received 9 June 2000)

\begin{abstract}
We report first-principles electronic-structure calculations using local density approximation in density functional theory for hexagonally bonded hetero-sheets $\mathrm{BNC}_{x}$ consisting of graphite and boron-nitride strips. We find a class of electron states that are localized at borders of the heterosheet. Calculations for other heterosheets and homosheets, $\mathrm{C}, \mathrm{BN}$, and $\mathrm{BC}_{3}$, clarify universal features of the border states, edge states, and nearly-freeelectron states. Electronic properties related to the border states in planer or tubular $\mathrm{BNC}_{x}$ are discussed.
\end{abstract}

Electron states in condensed matters are classified into several groups. In periodic systems they are labeled as Bloch states, whereas imperfection induces different classes of states: Point defects usually induce localized states in energy gaps of semiconductors or insulators; surfaces or interfaces are regarded as planer imperfections and localized electron states (surface/interface states) emerge near the twodimensional imperfection. These localized states are crucial to determine electronic properties of the materials.

Recently, an interesting class of localized states has been found in graphite ribbons (graphite sheets with finite sizes) which are expected to exist in growth processes of fullerenes ${ }^{1}$ or carbon nanotubes: ${ }^{2,3}$ A tight-binding ${ }^{4,5}$ and later the local density ${ }^{6}$ calculations show that some electron states at the Fermi level $\left(E_{\mathrm{F}}\right)$ are localized at but extended along an edge of the graphite ribbon (edge states), but that the edge states exhibit no dispersion in a direction along the edge in a part of Brillouin zone (Fig. 4 in Ref. 4). The edge state is peculiar to specific ribbons in which edges consist of zigzag arrangements of carbon atoms: Ribbons with edges consisting of armchair arrangements do not exhibit such edge states. ${ }^{4,5}$ It is generally shown that this class of localized states emerges in a system in which electron transfers among several atomic sites satisfy certain conditions. ${ }^{4,5,7}$

The edge states are not peculiar to carbon: As will be shown below, when boron and nitrogen atoms are arranged in a hexagonal network ( $h$-BN), similar edge states appear in the energy gap. Experimentally, synthesis of hexagonal networks of $\mathrm{B}, \mathrm{N}$, and $\mathrm{C}$ have been achieved both in planer and tubular forms. ${ }^{8-11}$ Experimental results ${ }^{9,11}$ and recent total energy calculations ${ }^{12}$ are indicative of phase separation of graphite and $h$-BN in $\mathrm{BNC}_{x}$ compounds. Although the atomic structure of the boundary in the separated phase is not clear yet, it is possible that there are borders between graphite and $h$-BN strips in phase-separated planer structures. These borders are expected to induce new electron states.

In this paper, we report first-principles calculations that clarify electronic structures of $\mathrm{BNC}_{x}$ hexagonal ribbons consisting of $h$-BN and graphite strips. We find that there are border states whose wave functions are localized at and extended along borders of $h$-BN and graphite, and that the border states show flat dispersion near $E_{\mathrm{F}}$ and hereby affect electronic properties substantially. The border state is not a simple extension of the edge states predicted in the past: Chemical variety at the border provides interesting variation in electronic structure. We also find that another class of electron states which are called nearly free electron (NFE) states, descendants of interlayer states in graphite, ${ }^{13}$ indeed exists on the $\mathrm{BNC}_{x}$ sheet and show a feature unexpected in the past.

All calculations have been performed by densityfunctional theory. ${ }^{14,15}$ Exchange-correlation energy of interacting electrons is treated in local density approximation (LDA) with a functional form fitted to the Ceperley-Alder result. ${ }^{16,17}$ Norm-conserving pseudopotentials generated by using the Troullier-Martins scheme are adopted to describe the electron-ion interaction. ${ }^{18,19}$ The valence wave functions are expanded by the plane-wave basis set with a cutoff energy of $50 \mathrm{Ry}$, which gives enough convergence of relative total energies of carbon-related materials ${ }^{18}$ and of $h$-BN. ${ }^{20}$ We adopt the conjugate-gradient minimization scheme both for the electronic-structure calculation and for the geometry optimization. ${ }^{21}$ Structural optimization has been performed until the remaining forces are less than $0.005 \mathrm{Ry} / \AA$. We use a repeating sheet model in which each atomic sheet is separated by $9.0 \AA$ to simulate a hexagonally bonded monolayer. Integration over Brillouin zone (BZ) is carried out using equidistant $k$-point sampling in which the $k$-point density is equivalent to the case of the 80 point sampling in the conventional $\mathrm{BZ}$ of graphite monolayer.

Figure 1 shows calculated energy bands of the $h$-BN ribbon in which edges have the zigzag shapes and are terminated with hydrogen. The ribbon is insulating with the calculated energy gap of $5 \mathrm{eV}$. The highest occupied (HO) and the lowest unoccupied (LU) bands show extremely flat dispersion around the zone boundary $X$. The wave functions of the HO and LU bands clearly show that these states are edge states whose wave functions are localized at one of edges. Due to chemical difference of B and N, the LU edge state is localized at the edge of B atoms, whereas the $\mathrm{HO}$ edge state is localized at the edge of $\mathrm{N}$ atoms. These edge states are unable to be derived from zone folding analysis of energy bands of the $h$-BN monolayer. ${ }^{22}$ They emerge due to subtle balance of electron transfers among $\pi$ orbitals situated at edge atoms, hereby exhibiting the peculiar flat band nature. These features have been also observed for the edge states in graphite ribbon with the zigzag edges. ${ }^{4,6}$ Hence it is now clarified that the edge state is a common product in hexagonally networked ribbons with zigzag shaped edges. More 
(a)
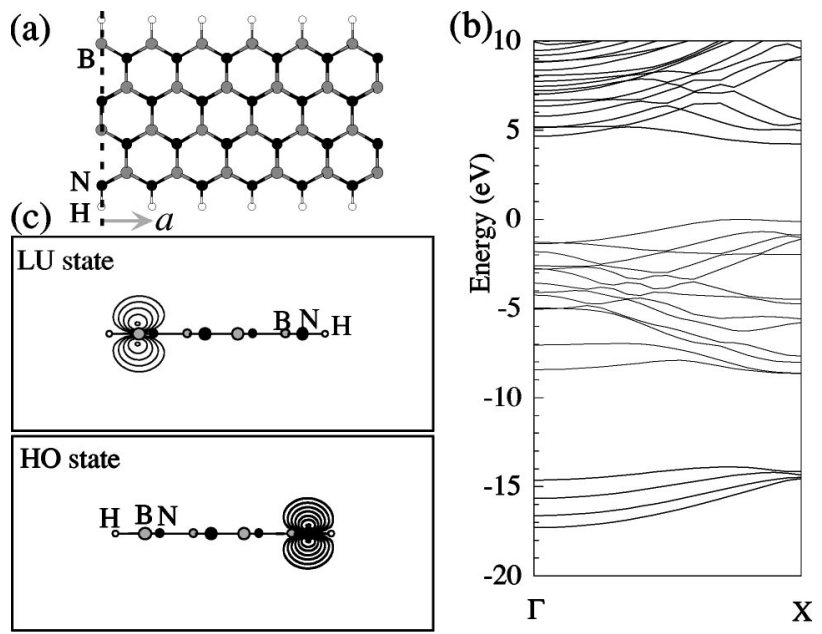

FIG. 1. (a) Top view of $h$-BN ribbon with infinite length along the vector $a$. Black, dark, and white circles denote $\mathrm{N}, \mathrm{B}$, and $\mathrm{H}$ atoms, respectively. (b) Energy bands of the H-terminated $h$-BN ribbon. The direction $\Gamma X$ is parallel to the vector $a$ in (a). Energy is measured from the top of occupied bands. (c) Contour plots of the squared wave functions of the LU and the $\mathrm{HO}$ states at $X$ point represented on the cross section of the ribbon containing the dashed line $\mathrm{BN}$ in (a). Each contour represents twice (or half) the density of its adjacent lines. The highest values are $1.21 / \AA^{3}$ and $3.37 / \AA^{3}$ for the LU and the HO states, respectively.

generally, even the edges are not required. Zigzag shaped borders are enough to produce the peculiar localized states, as is shown below.

Figure 2 shows energy bands of $\mathrm{BNC}_{2}, \mathrm{BNC}_{4}$, and $\mathrm{BNC}_{6}$ sheets in which $h$-BN and graphite strips are separated and the borders are of zigzag shapes. We have found that these sheets are semiconducting with the direct energy gaps and that the gap energy monotonically decreases with increasing the width of graphite strips. We find that the highest occupied $\pi$ and the lowest unoccupied $\pi^{*}$ bands show almost flat dispersion around the zone boundary $J$. This feature is similar to that of the energy bands of hexagonally networked ribbons described above.

In order to clarify characteristics of the flat band states in the $\mathrm{BNC}$ sheets, we calculate their wave functions in $\mathrm{BNC}_{4}$ (Fig. 3). It is found that the highest $\pi$ state at $J$ (labeled CB in Fig. 2) is localized at $\mathrm{C}$ and $\mathrm{B}$ atoms which constitute one of borders of $h$-BN and graphite strips. The state has bonding $\pi$ character with substantial hybridization of $\pi$ orbitals of C and $\mathrm{B}$ atoms. It is then naturally expected that the antibonding state also exists. We find that a state in the conduction bands has such character (CB* in Fig. 2). The wave function of $\mathrm{CB}^{*}$ shown in Fig. 3(a) is located at the border and clearly manifests its antibonding character. The wave function of the lowest conduction band at $J\left(\mathrm{CN}^{*}\right.$ in Fig. 2) is also shown in Fig. 3(a). It is localized at $\mathrm{C}$ and $\mathrm{N}$ atoms in this case which constitute the other border of $h$-BN and graphite strips. Again substantial hybridization of $\pi$ orbitals of $\mathrm{C}$ and $\mathrm{N}$ atoms is observed. The state has antibonding character. We have explored the valence bands and found a state labeled $\mathrm{CN}$. The wave function of $\mathrm{CN}$ is located along the border formed by $\mathrm{C}$ and $\mathrm{N}$ and is of bonding character [Fig. 3(a)]. It is now clarified that zigzag borders of hexagonally networked heterosheets induce a new class of electron states which are localized along the borders: the border states.

It is emphasized that the states are peculiar to the zigzag borders. Figures $4(\mathrm{a})$ and $4(\mathrm{~b})$ show the geometry and the electronic band structure of $\mathrm{BNC}_{2}$ sheet where the borders between graphite and $h$-BN strips are of armchair shape. We find that the system possesses direct energy gap at $\Gamma$ point and the highest occupied and the lowest unoccupied states around the $\Gamma$ point do not show the flat band nature. The characteristics of the band structure is completely different from those of the heterosheet with zigzag borders. The corresponding wave functions of the states at $\Gamma$ point are not localized at the border but extended over the whole sheet [Fig. 4(c)]. In this case, the border states are absent in spite of the presence of the borders between different species of atoms.

We also find that the highest occupied $\pi$ and the lowest unoccupied $\pi^{*}$ states lose their border-state character with decreasing wave numbers: The wave functions of the states at $\Gamma$ point $\left[\Gamma_{\mathrm{HP}}\right.$ and $\Gamma_{\mathrm{LP}} *$ in Fig. 2(b) and Fig. 3(c)] is extended over the whole $\mathrm{BNC}_{4}$ heterosheet [Fig. 3(c)]. Consequently the total valence electron density is also extended over the sheet [Fig. 3(d)]. The border states we have found are therefore extremely unique and different from the usual interface states which maintain their localized character in whole BZ.

The electronic structures of $\mathrm{BNC}_{x}$ described above have been obtained after complete optimization of atomic geometries. The calculated bond length of $\mathrm{C}-\mathrm{N}$ and $\mathrm{B}-\mathrm{C}$ at the border is 1.37 and $1.50 \AA$, respectively. The length of C-C

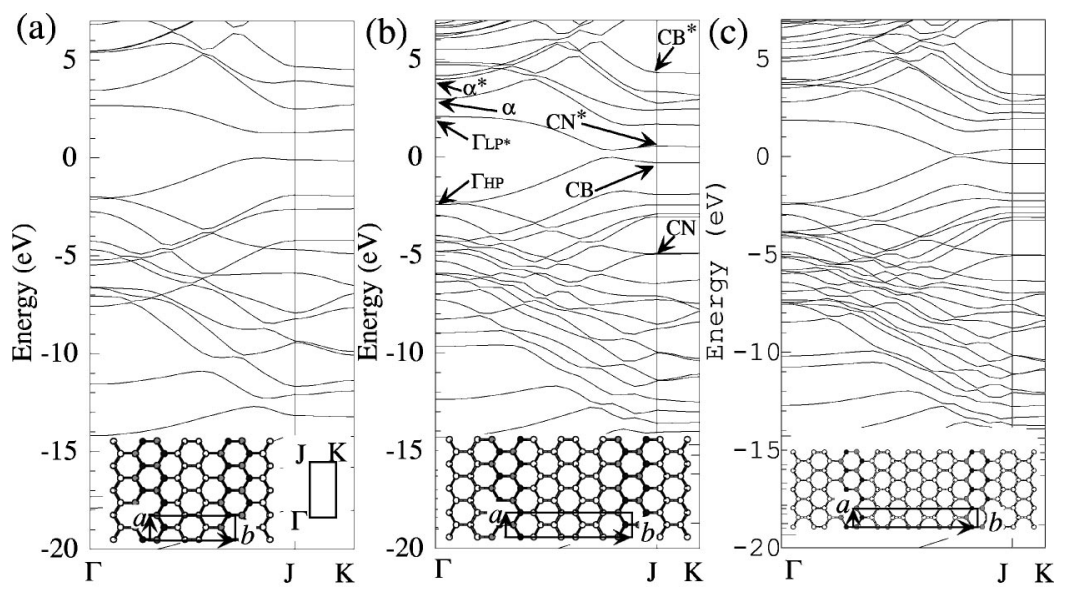

FIG. 2. Energy bands of (a) $\mathrm{BNC}_{2}$, (b) $\mathrm{BNC}_{4}$, and $(\mathrm{c}) \mathrm{BNC}_{6}$ sheets. A part of each sheet is shown in each inset where white, black, and shaded circles are $\mathrm{C}, \mathrm{N}$, and $\mathrm{B}$ atoms, respectively. Periodicity in each sheet is represented by vectors $a$ and $b$. Symmetry lines in twodimensional BZ is shown in the inset. The origin of the energy is the top of the occupied bands. See text for labels in (b) used for some bands at the BZ boundary and the center. 
(a)

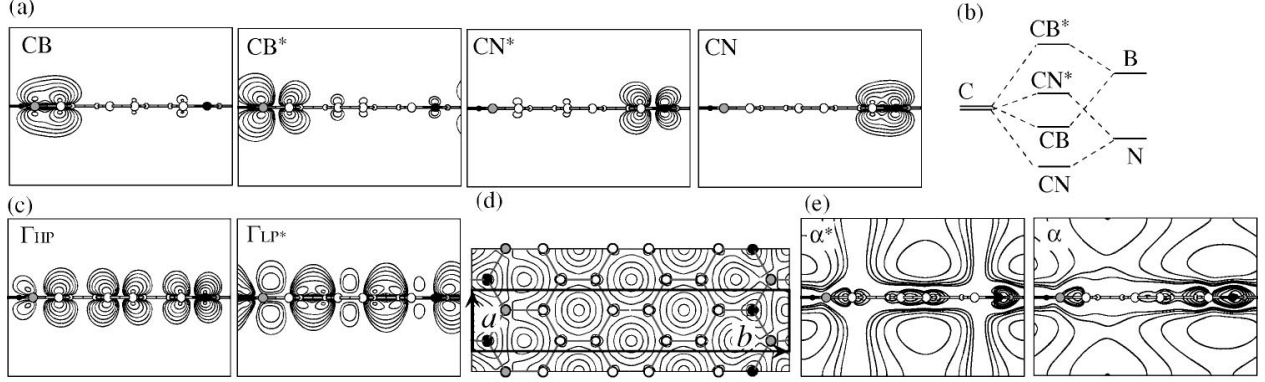

FIG. 3. Wave functions, charge density, and energy diagram of $\mathrm{BNC}_{4}$. (a) Contour plots of squared wave functions of the flat-band states of $\mathrm{BNC}_{4}$ at $J$, which are labeled as $\mathrm{CB}, \mathrm{CB}^{*}, \mathrm{CN}^{*}$, and $\mathrm{CN}$ in Fig. 2(b). Plots are on the vertical cross section of the sheet which contains the vector $b$ in (d) or in Fig. 2(b). White, black, and shaded circles denote C, N, and B atoms, respectively. (b) Schematic energy diagram for the hybridization of the edge states of graphite and of $h-\mathrm{BN}$ in $\mathrm{BNC}_{4}$ sheets. (c) Contour plots of squared wave functions of the highest occupied $\pi$ and the lowest unoccupied $\pi^{*}$ states at the zone center $\Gamma$. Plots are on the same cross section as in (a). (d) Contour plots of total valence charge density shown on the plane including the $\mathrm{BNC}_{4}$ sheet. (e) Contour plots of squared wave functions of NFE states at $\Gamma$, $\alpha$, and $\alpha^{*}$ (see Fig. 2 for labels) shown on the same vertical cross section as in (a). In the direction perpendicular to this paper, the states have NFE character. In all contour plots, each contour line represents twice (or half) the density of its adjacent contour lines. The lowest contours represent $2.11 \times 10^{-2} / \AA^{3}, 4.22 \times 10^{-3} / \AA^{3}, 2.11 \times 10^{-1} / \AA^{3}$, and $1.05 \times 10^{-3} / \AA^{3}$, for (a), (c), (d), and (e), respectively.

bonds is $1.40-1.42 \AA$ and that of $\mathrm{B}-\mathrm{N}$ is 1.40 and $1.43 \AA$. The bond lengths of $\mathrm{C}-\mathrm{C}$ and $\mathrm{B}-\mathrm{N}$ are similar to those in graphite and $h-\mathrm{BN}$. The heterobonds, B-C and $\mathrm{N}-\mathrm{C}$, on the other hand, are less strong than the $\mathrm{C}-\mathrm{C}$ or $\mathrm{B}-\mathrm{N}$ bonds. Hence the phase separation leading to the striped structures of the $h$-BN and graphite discussed above is energetically favorable.

Figure 3(b) shows an energy diagram of the edge states of the ribbons hybridized in the heterosheet: The edge states of graphite ribbon and of the $h$-BN ribbon are hybridized at the borders in $\mathrm{BNC}_{4}$ heterosheet, becoming the four border states. Due to the energy-level difference of three $\pi$ orbitals of $\mathrm{C}, \mathrm{N}$, and $\mathrm{B}$ atoms, the four border states are in the order of $\mathrm{CN}, \mathrm{CB}, \mathrm{CN}^{*}$, and $\mathrm{CB}^{*}$ in increasing energy, and the two middle states appear near $E_{\mathrm{F}}$. The borders consisting of different atom species introduce different energy levels and then the hybridization renders the energy gap open at $J$.

This situation becomes more prominent in other hexagonally networked heterosheets with zigzag borders. $\mathrm{BC}_{3}$ compounds have also been synthesized and the structure has hexagonal symmetry. ${ }^{23}$ In this case, we also find that the border states are localized on the zigzag border of $\mathrm{B}$ and $\mathrm{C}$ atoms and the states render the flat bands at the Fermi level. On the contrary to the $\mathrm{BNC}_{x}$ sheet, $\mathrm{BC}_{3}$ sheet shows no energy gap. Subtle balance of electron transfers among atomic sites, that is achieved at the zigzag borders, induces the unique border states described above. The subtle balance is not specific but general in certain atomic arrangements. The latter infers that introduction of chemical variety at the border enriches the border state both in distribution of its wave function and in location of its energy level.

As stated above, the highest occupied $\pi$ and the lowest unoccupied $\pi^{*}$ states of the heterosheet $\mathrm{BNC}_{x}$ are the border states in character around the zone boundary $J$, whereas they are extended over the sheet around the zone center $\Gamma$. This implies potential applicability of $\mathrm{BNC}_{x}$ compounds. For instance, the striped $\mathrm{BNC}_{x}$ compound is capable of modulating the carrier flow by using the border states as channels of conduction. Carriers injected into the compound are first accommodated in either the highest $\pi$ or the lowest $\pi^{*}$ bands at $J$. The injected carriers are thus of one-dimensional char- acter along the borders. Then by exciting carriers or by increasing the number of carriers, the states around $\Gamma$ become occupied. In this case, the conduction is isotropic reflecting the character of the wave function.

Unusual distribution of the wave function that we have found is also utilized for nanocoils when $\mathrm{BNC}_{x}$ is formed in a tubular form. Upon relatively light carrier doping, the current is one-dimensional along the border of the graphite and the $h$-BN strips. Therefore by tuning the width ratio of the strips and by forming appropriate tubular structure, we can control chirality of the current along the $\mathrm{BNC}_{x}$ tube. This is a generalization of the chiral conductivity first proposed for the $\mathrm{BNC}_{2}$ tube ${ }^{24}$ where anisotropic conductivity of the $\mathrm{BN}$ monolayer is utilized. As a limiting case, the current flow in the $\mathrm{BNC}_{x}$ tube consisting of the $h-\mathrm{BN}$ and the graphite strips (a)

(c)

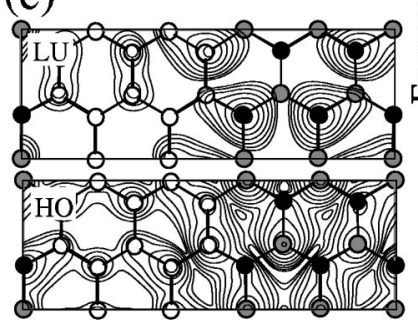

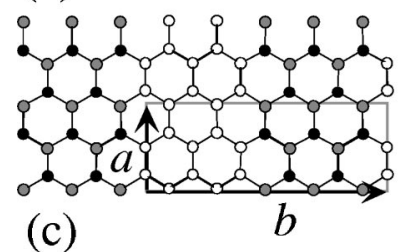

(b)

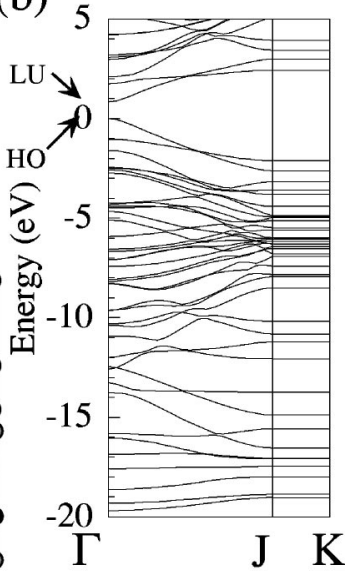

FIG. 4. (a) Geometric structure of $\mathrm{BNC}_{2}$ heterosheet where the borders are of armchair shape. White, black, and shaded circles denote $\mathrm{C}, \mathrm{N}$, and $\mathrm{B}$ atoms, respectively. (b) Energy band of the striped $\mathrm{BNC}_{2}$ heterosheet. The origin of the energy is the top of the occupied band denoted by HO. (c) Contour plots of squared wave function of the highest occupied (HO) and the lowest unoccupied (LU) states at $\Gamma$ point. In both contour plots, each contour line represents twice (or half) the density of its adjacent contour line. The lowest contour represents $4.22 \times 10^{-3} / \AA^{3}$. 
with its index (n, 0) (zigzag tube) $)^{3}$ is completely circular around the tube, being regarded as a nanosolenoid.

The borders of striped $\mathrm{BNC}_{x}$ also cause interesting modification of wave functions of nearly-free-electron (NFE) states. The NFE state or the interlayer state exists above $E_{\mathrm{F}}$ in the case of graphite monolayer ${ }^{13}$ and as the LU state in BN monolayer. ${ }^{22}$ Figure 3(e) shows the wave functions of the NFE states $\alpha$ and $\alpha^{*}$ which are located above $E_{\mathrm{F}}$ (Fig. 2) in $\mathrm{BNC}_{4}$. The energy levels of the NFE states in monolayer graphite and $\mathrm{BN}$ are different from each other. The borders in $\mathrm{BNC}_{4}$ induce hybridization of the two different NFE states. As a result, we find the bonding and antibonding NFE states clearly shown in Fig. 3(e): The bonding state $\alpha$ has more amplitude on the $\mathrm{BN}$ strip, whereas the antibonding state $\alpha^{*}$ does on the graphite strip. Also the $\alpha^{*}$ state has nodes along the borders, whereas both $\alpha$ and $\alpha^{*}$ preserve their NFE character along the strips.

In conclusion, we have presented LDA calculations which clarify unique and interesting features of the border states inherent to hexagonally networked heterosheets.

Computations were performed at ISSP, University of Tokyo and at IMS (Okazaki). This work was supported in part by JSPS under Contract No. RFTF96P00203 and the Grantin-Aid for Scientific Research Grant No. 11740219 and Grant No. 10309003.
${ }^{1}$ H. Kroto et al., Nature (London) 318, 162 (1985); W. Krätschmer et al., ibid. 347, 354 (1990).

${ }^{2}$ S. Iijima, Nature (London) 354, 56 (1991).

${ }^{3}$ N. Hamada, S. Sawada, and A. Oshiyama, Phys. Rev. Lett. 68, 1579 (1992); J.W. Mintmire, B.I. Dunlap, and C.T. White, ibid. 68, 631 (1992); R. Saito et al., Appl. Phys. Lett. 60, 2204 (1992).

${ }^{4}$ M. Fujita et al., J. Phys. Soc. Jpn. 65, 1920 (1996).

${ }^{5}$ K. Nakada et al., Phys. Rev. B 54, 17954 (1996).

${ }^{6}$ Y. Miyamoto, K. Nakada, and M. Fujita, Phys. Rev. B 59, 9858 (1999).

${ }^{7}$ Y. Takagi et al., submitted to Phys. Rev. B (to be published). Earlier example of the flat band and its ferromagnetism is reviewed by H Tasaski, Prog. Theor. Phys. 99, 489 (1998).

${ }^{8}$ M. Kawaguchi and T. Kawashima, J. Chem. Soc. Chem. Commun. 1133 (1993); O. Stephan et al., Science 266, 1683 (1994); Z. Weng-Sieh et al., Phys. Rev. B 51, 11229 (1995).

${ }^{9}$ K. Suenaga et al., Science 278, 653 (1997); Y. Zhang et al., Chem. Phys. Lett. 279, 264 (1997).

${ }^{10}$ M.O. Watanabe et al., Phys. Rev. Lett. 77, 187 (1996).

${ }^{11} \mathrm{Ph}$. Kohler-Redlich et al., Chem. Phys. Lett. 310, 459 (1999).

${ }^{12}$ X. Blase et al., Appl. Phys. A: Mater. Sci. Process. 68, 293
(1999).

${ }^{13}$ M. Posternak et al., Phys. Rev. Lett. 50, 761 (1983).

${ }^{14}$ P. Hohenberg and W. Kohn, Phys. Rev. 136, B864 (1964).

${ }^{15}$ W. Kohn and L.J. Sham, Phys. Rev. 140, A1133 (1965).

${ }^{16}$ D.M. Ceperley and B.J. Alder, Phys. Rev. Lett. 45, 566 (1980).

${ }^{17}$ J.P. Perdew and A. Zunger, Phys. Rev. B 23, 5048 (1981).

${ }^{18}$ N. Troullier and J.L. Martins, Phys. Rev. B 43, 1993 (1991).

${ }^{19}$ L. Kleinman and D.M. Bylander, Phys. Rev. Lett. 48, 1425 (1982).

${ }^{20}$ Convergence of the total energy with respect to the cutoff energy is examined on the $h$-BN by performing the computation with 30, 40, 50, 60, and 80 Ry. The total energy obtained with 50 Ry is larger by $0.02 \%$ than that obtained with $80 \mathrm{Ry}$. The lattice constant computed with the cutoff energy 50 Ry is $a$ $=2.475 \AA$ which is $1.16 \%$ smaller than the experimental value. Sufficient convergence of energy bands is obtained by smaller cutoff energies.

${ }^{21}$ O. Sugino and A. Oshiyama, Phys. Rev. Lett. 68, 1858 (1992).

${ }^{22}$ X. Blase et al., Phys. Rev. B 51, 6868 (1995).

${ }^{23}$ B.M. Way et al., Phys. Rev. B 46, 1697 (1992).

${ }^{24}$ Y. Miyamoto, S.G. Louie, and L. Cohen, Phys. Rev. Lett. 76, 2121 (1996). 